\title{
Global climate goes regional, and vice versa: Reflecting on 14 years of the PAGES $2 \mathrm{k}$ Network
}

\author{
Nerilie Abram¹, D. Kaufman², H. McGregor ${ }^{3}$, B. Martrat ${ }^{4}$, O. Bothe ${ }^{5}$ and H. Linderholm ${ }^{6}$

\begin{abstract}
For the past 14 years, the PAGES 2k Network has brought together a large, interdisciplinary community to better understand pre-industrial climate and give context to recent human-caused climate change.
\end{abstract}

The past 2000 years of Earth's history provides a critical context for understanding climate variability and change. This is a period where climate changes occurred as a result of well characterized natural climate forcing, as well as unforced internal variability, and has now transitioned into a climate that is strongly forced by human factors. It is also a period where a range of paleoclimate proxy records, often with annual or better resolution, can be used to build up a comprehensive spatial understanding of our climate system. Recent step changes in computing capabilities now allow for ensembles of millennial-length climate-model simulations with which researchers can test and improve our knowledge of the climate system. All of these factors provide a rich scientific backdrop for the work of the PAGES $2 k$ Network.

The $2 k$ Network is one of the longest running working groups of PAGES. Now in its 14 th year, the $2 \mathrm{k}$ Network has generated more than 54 journal articles, two major paleoclimate databases for temperature and hydrology reconstructions, and around 40 Past Global Changes Magazine articles, while fostering an open and collaborative work environment with an emphasis on FAIR data stewardship principles (Wilkinson et al. 2016).

\section{Evolution of the 2k Network}

When the 4th Assessment Report of the Intergovernmental Panel on Climate Change was released in 2007, it stated that "Palaeoclimate information supports the interpretation that the warmth of the last half century is unusual in at least the previous 1,300 years" (IPCC 2007, p. 9). This was a finding that specifically related to the Northern Hemisphere, due to the low density of available proxy records from the Southern Hemisphere and tropics, and where questions remained over statistical reconstruction methods and the suitability of different proxy records.

A Past Global Changes Magazine article in 2005 highlighted the opportunities that dense networks of high-quality natural and documentary archives offer for moving beyond global average, or Northern Hemisphere average, temperature reconstructions (Wanner 2005) to resolve spatial patterns of climate variation. This effort coincided with an interest in testing the increasingly smaller-scale climate information being simulated by new generations of climate models. Wanner posited that "the complex dynamical processes leading to past and future climate and environmental change can only be understood if we also acquire insight into the regional dynamics." This, through the LOTRED (Long-Term Climate Reconstruction and Dynamics) approach, set in motion the beginnings of the PAGES $2 k$ Network.

The first phase began in 2008, with the goal of assembling paleoclimate records over specific regions and using these to produce continent-scale temperature reconstructions. The work initially involved eight regional working groups covering each continent and its surroundings (including an Arctic2k group). Later, an Ocean2k working group was also established to collate paleoclimate data from the world's oceans. Each group used their own expertise to assess the suitability of paleoclimate records and the best methods for combining these proxies into continent- or ocean-basin-scale temperature reconstructions.

The second phase involved trans-regional projects that brought together the datasets compiled across the regional working groups to answer specific scientific questions about the timing and inter-hemispheric variability of natural and anthropogenic climate changes over the past 2000 years. Phase 2 also included efforts to unify and test the statistical methods of reconstructing climate, and assembled a global paleoclimate temperature database with best practices of data management and accessibility. Work of the regional working groups

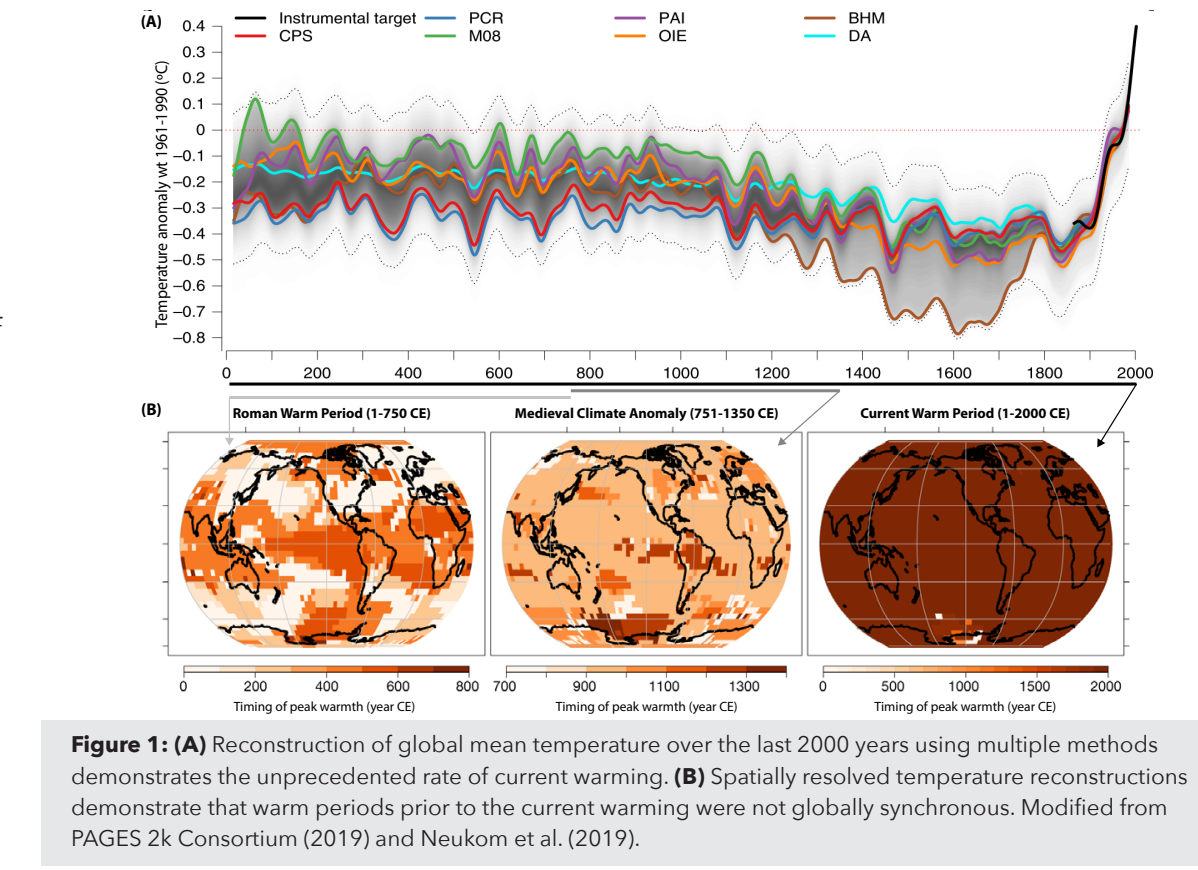

Figure 1: (A) Reconstruction of global mean temperature over the last 2000 years using multiple methods demonstrates the unprecedented rate of current warming. (B) Spatially resolved temperature reconstructions demonstrate that warm periods prior to the current warming were not globally synchronous. Modified from PAGES 2k Consortium (2019) and Neukom et al. (2019).

also continued with efforts to develop hydroclimate reconstructions and resolve spatial patterns in climate changes. A major product of Phase 2 was a special issue of Climate of the Past titled "Climate of the past 2000 years: regional and trans-regional syntheses". This special issue also focused on putting into practice open-access data stewardship principles (Kaufman et al. 2018).

Phase 3 of the PAGES 2k project has seen the conclusion of some trans-regional projects as they achieved their goals, and the development of new project groups under the $2 \mathrm{k}$ Network banner. These project groups are community-led projects that are working towards the Phase 3 goals to: (1) build further understanding of climate variability, modes and mechanisms, (2) improve reconstruction methods and reduce uncertainties, and (3) assess proxy-model agreement.

\section{Highlights of $\mathbf{2 k}$ Network research}

The first major synthesis of the PAGES $2 k$ Network showed that across seven recon structed continental regions, all experienced a long-term cooling trend during pre-industrial times over the past 2000 years (PAGES 2k Consortium 2013). Long-term cooling of the global ocean also characterized the last 2000 years (McGregor et al. 2015; Tierney et al. 2015). Reconstructed long-term preindustrial cooling is robust across different reconstruction methods, is consistent with last millennium climate model simulations, especially for the Northern Hemisphere 

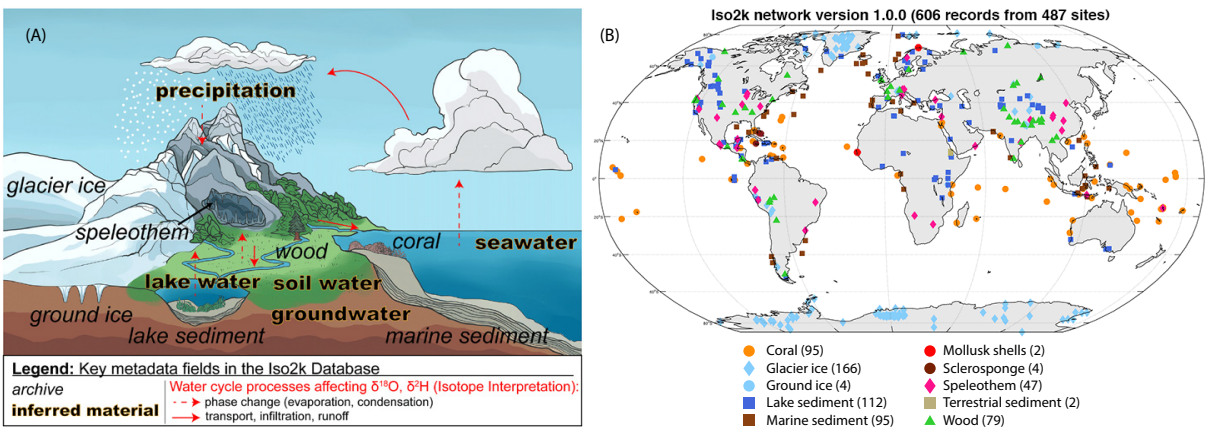

Figure 2: (A) Schematic illustration of the global water cycle and key metadata fields in the Iso2k database, and (B) spatial distribution of isotope records in the Iso2k database. Adapted from Konecky et al. (2020).

(PAGES 2k-PMIP3 group 2015), and is thought to be largely a response to accumulated volcanic forcing of pre-industrial climate (McGregor et al. 2015). Multidecadal fluctuations over the past 2000 years have a coherent magnitude and timing between global temperature reconstructions and appear to also be attributable to volcanic forcing during pre-industrial times (PAGES $2 k$ Consortium 2019). Spatial reconstructions of this temperature variability have now found that there were no warm or cold periods during pre-industrial times of the past two millennia that occurred at a global scale (Neukom et al. 2019; Fig. 1).

The onset of industrial-era warming began in the mid to late 19th century in all ocean and land areas except Antarctica (Abram et al. 2016), i.e. earlier than can be assessed based on historical observations alone.

Sustained warming began first over Northern Hemisphere land masses and in the tropical oceans, and was delayed in the Southern Hemisphere possibly due to Southern Ocean circulation processes, but this delayed southern warming onset is not currently reproduced by climate, model simulations. The 20th century warming interval is the only time in the past millennium when both hemispheres have experienced contemporaneous warm extremes (Neukom et al. 2014), and the second half of the 20th century has the largest global warming trends (at timescales of 20 years or longer) of any time in the past 2000 years - highlighting the unprecedented character of recent human-caused warming compared with natural climate variability in the past (PAGES $2 \mathrm{k}$ Consortium 2019).

The work behind these research highlights has led to, or been enabled by, the most well documented and extensive database of temperature sensitive proxies of the past 2000 years published to date (PAGES 2k Consortium 2017). The community-sourced database gathered 692 records from 648 locations, including all continental regions and major ocean basins, and is shared in the Linked Paleo Data (LiPD) format (McKay and Emile-Geay 2016) with options provided for accessing the database in multiple coding languages. A similar effort has now also resulted in a comprehensive global database of water isotope $\left(\delta^{18} \mathrm{O}\right.$ and $\left.\delta \mathrm{D}\right)$ proxies for investigating variability and trends in global hydroclimate (Konecky et al. 2020; Fig. 2).

Ongoing work within the $2 \mathrm{k}$ Network seeks to resolve natural versus anthropogenic trends in the global hydrological cycle, and linkages between the marine hydrological cycle and the terrestrial hydrological cycle. Several reconstructions have emerged over the past few years that shed new light on regional hydroclimate, including precipitation in Antarctica (Thomas et al. 2017) and Australia (Freund et al. 2017), the evolution of the Southern Annular Mode and its teleconnections (Dätwyler et al. 2018), and drought in Scandinavia (Seftigen et al. 2017). Regional studies also highlighted the problem of spatial gaps in hydroclimate data, which are obvious in the Southern Hemisphere (Nash et al. 2016; Gergis and Henley 2017), as well as parts of the Northern Hemisphere, including the Arctic (Linderholm et al. 2018). Others highlighted the importance of historical documents for our understanding of past hydroclimatic changes and their societal impacts (GuevaraMurua et al. 2018; Gil-Guirado et al. 2019).

A framework has been established for comparing modeled and reconstructed estimates of past hydroclimates in order to quantitatively constrain future hydroclimate risk (PAGES Hydro2k Consortium 2017).

Proxy data-model comparisons have shown that northern hemispheric paleodata do not support the intensification of 20th century wet and dry anomalies produced by models (Ljungqvist et al. 2016), and paleodata from Europe further suggest that model simulations may overestimate the risk of temperature-driven droughts in Europe (Ljungqvist et al. 2019). Hydroclimatic perspectives on the Common Era have also been put in a longer context, demonstrating the potential for current weakening of the latitudinal temperature gradient in the Northern Hemisphere to reduce mid-latitude rainfall (Routson et al. 2019).

\section{Future of the 2k Network}

Discussions are underway within the community (PAGES 2k Network coordinators 2020) over the future of $2 \mathrm{k}$ activities after Phase 3 wraps up at the end of 2021. Throughout the history of the $2 k$ Network, the scientific endeavors have been driven by an organic, grassroots approach. Individuals, often early-career researchers, have brought their ideas, enthusiasm, and leadership to the different activities. As specific projects have been completed, others have sprung up. As researchers have moved on to other priorities, others have joined and renewed the activities and direction of the $2 \mathrm{k}$ effort. The $2 \mathrm{k}$ Network has produced ground-breaking science, while also building scientific careers and fostering collaborations across an international scientific community.

It is clear that there is momentum within some of the current $2 \mathrm{k}$ projects that will continue to yield valuable scientific outcomes beyond 2021. Other $2 \mathrm{k}$ projects with important ambitions are still in the early phases. Over the coming months the $2 k$ coordinators will continue the efforts already begun and work with the research community to develop a plan for future $2 \mathrm{k}$ research priorities. These include the curation of data products as well as the rescue of existing data sources, building stable bridges between the paleodata and paleomodeling communities, a more holistic $2 \mathrm{k}$ view of the climate system that goes beyond temperature, and, finally, using $2 \mathrm{k}$ research to provide information that aids society and guides policy decisions.

\section{AFFILIATIONS}

${ }^{1}$ Research School of Earth Sciences, Australian National University, Canberra, Australia ${ }^{2} S c h o o l ~ o f$ Earth and Sustainability, Northern Arizona University, Flagstaff, USA

${ }^{3}$ School of Earth and Environmental Sciences, University of Wollongong, Australia ${ }^{4}$ Institute of Environmental Assessment and Water Research (IDAEA-CSIC), Barcelona, Spain ${ }^{5}$ Helmholtz-Zentrum Hereon, Geesthacht, Germany ${ }^{6}$ Department of Earth Sciences, University of Gothenburg, Sweden

\section{CONTACT}

Nerilie Abram: nerilie.abram@anu.edu.au

\section{REFERENCES}

Abram N et al. (2016) Nature 536: 411-418 Dätwyler et al. (2018) Clim Dyn 51: 2321-2339 Freund M et al. (2017) Clim Past 13: 1751-1770 Gergis J, Henley B (2017) Clim Dyn 48: 2087-2105 Gil-Guirado S et al. (2019) Clim Past 15: 1303-1325 Guevara-Murua A. (2018) Clim Past 14: 175-191 IPCC (2007) In: Solomon S et al. (Eds) Climate Change 2007: The Physical Science Basis. Cambridge University Press, 1-18

Kaufman D et al. (2018) Clim Past 14: 593-600

Konecky B et al. (2020) Earth Syst Sci Data 12: 2261-2288

Linderholm H et al. (2018) Clim Past 14: 473-514

Ljungqvist F et al. (2016) Nature 532: 94-98 Ljungqvist F et al. (2019) Environ Res Lett 14: 084015 McGregor H et al. (2015) Nat Geosci 8: 671-677 McKay N, Emile-Geay J (2016) Clim Past 12: 1093-1100 Nash D et al. (2016) Quat Sci Rev 154: 1-22 Neukom R et al. (2014) Nat Clim Change 4: 362-367 Neukom R et al. (2019) Nature 571: 550-554 PAGES 2k Consortium (2013) Nat Geosci 6: 339-346 PAGES 2k Consortium (2017) Sci Data 4: 170088

PAGES 2k Consortium (2019) Nat Geosci 12: 643-649 PAGES 2k Network coordinators (2020) PAGES Mag 28: 66 PAGES 2k-PMIP3 group (2015) Clim Past 11: 1673-1699 PAGES Hydro2k Consortium (2017) Clim Past 13: 1851-1900

Routson C et al. (2019) Nature 568: 83-87 Seftigen Ket al. (2017) Clim Past 13: 1831-1850 Thomas ER et al. (2017) Clim Past 13: 1491-1513 Tierney JE et al. (2015) Paleoceanography 30: 226-252 Wanner H. (2005) PAGES news 13: 19-21

Wilkinson M et al. (2016) Sci Data 3: 160018 